\title{
Cobertura de úlceras por presión trocantéricas con colgajo de tensor de fascia lata en $\mathrm{V}-\mathrm{Y}^{*}$
}

\author{
Drs. WILFREDO CALDERÓN O. ${ }^{1}$, EDUARDO OYARSE M. ${ }^{1}$, CAROLINA OLIVARES G. ${ }^{1}$, \\ HÉCTOR ROCO M. ${ }^{1}$, PATRICIO LENIZ M. ${ }^{1}$, DANIEL CALDERÓN M. ${ }^{1}$, \\ HERNÁN NORAMBUENA B. ${ }^{1}$, FERNANDA DEICHLER V. ${ }^{1}$ \\ 1 Servicio de Cirugía Plástica y Quemados. Hospital del Trabajador. Santiago, Chile.
}

\begin{abstract}
Treatment of trochanteric pressure sores with $\mathrm{V}-\mathrm{Y}$ and tensor fasciae latae flaps

Background: Trochanter pressure sores can be treated with random, myocutaneous, fasciocutaneous or free flaps. Aim: To report the use of V-Y and tensor fasciae latae flaps for the treatment of trochanteric pressure sores. Material and Methods: Prospective study of 14 males and two females aged 24 to 64 years, with 17 sores. The surgical technique consisted in the design of a V shaped flap with irrigation in its superior base and lower vertex with one of its extremes in contact with the sore. A resection is made until a vital base is obtained, excising the prominent bone until a plane in which healthy tissue is seen. The flap is raised and rotated, covering the defect. A V-Y primary closure without tension is performed, leaving drainages that are withdrawn ten days later. Results: The complications recorded were three seromas, which were treated using a closed system with external and internal negative pressure. Two wound dehiscence episodes and two hematomas required a surgical correction. Conclusions: This type of flap is other therapeutic alternative for pressure trochanteric sores.
\end{abstract}

Key words: Trochanteric sores, surgical flaps, pressure ulcers.

\section{Resumen}

Las úlceras por presión trocantéricas representan un problema importante a nivel extra e intrahospitalario. Existen múltiples opciones terapéuticas, ya sean colgajos random, musculocutáneos, fasciocutáneos o libres. El objetivo de este estudio es presentar la técnica quirúrgica del Colgajo en V-Y de Tensor de Fascia Lata. Técnica Quirúrgica: Se diseña un colgajo en V con irrigación por su base superior y vértice inferior, contactando uno de sus extremos con la úlcera. La forma del colgajo es de una V-Y. Se reseca esta hasta obtener un lecho vital, resecando además el hueso prominente hasta un plano en que se visualiza tejido sano. Se levanta y rota el colgajo, cubriendo el defecto. Finalmente, se realiza el cierre primario en VY sin tensión, con drenajes que se retiran a los 10 días. Tratamos 17 úlceras en 16 pacientes. Hubo 3 seromas, los que fueron resueltos con sistema de cierre con presión negativa externa e interna. Además hubo 2 casos de dehiscencia de sutura y 2 hematomas resueltos en quirófano. Este colgajo debe estar presente dentro de las posibilidades terapéuticas que se ofrezcan a pacientes con úlceras trocantéricas por decúbito.

Palabras clave: Colgajo, úlcera por presión trocantérica.

*Recibido el 2 de Mayo de 2009 y aceptado para publicación el 29 de Mayo de 2009.

Correspondencia: Dr. Wilfredo Calderón O.

Ramón Carnicer 185 - 5º Piso, Santiago, Chile. Fax: 685.34.58

E-mail: wcalderón@hts.cl 


\section{Introducción}

La úlcera por presión (UPP) definida como lesión isquémica de tejidos blandos secundaria a la presión ejercida por una prominencia ósea, es una patología prevalente en los pacientes con síndrome de postración por lesión neurológica, hospitalización prolongada y pacientes añosos. Hasta el 50\% de las UPP se presentan en pacientes de 70 años o más.

La localización más frecuente corresponde a las úlceras trocantéricas, (20 al 34\%) y ocurre en paciente para y tetrapléjicos ${ }^{1}$.

En 1978 Nahai describió el colgajo tipo I de tensor de fascia lata ${ }^{2}$ para el tratamiento de las úlceras por presión trocantéreas. Sin embargo, la rotación posterior del colgajo predispone a formación de la 'oreja de perro'. Además, el cierre del sitio donante generalmente es bajo tensión, lo que predispone a la dehiscencia y necrosis en el post operatorio. Se han propuesto múltiples modificaciones de la técnica, buscando disminuir la tensión de la línea de sutura, mejorar la estética y el resultado final ${ }^{3-5}$. En nuestra casuística estos objetivos se cumplen realizando un colgajo de tensor de la fascia lata en V$\mathrm{Y}$ (avance en $\mathrm{V}$ y cierre en $\mathrm{Y}$ ).

El objetivo de este estudio es presentar y analizar las características del Colgajo de Tensor de Fascia Lata, para úlceras por presión trocantéricas realizadas en el Hospital del Trabajador de Santiago. Con esta metódica de colgajo se logra minimizar la tensión en la punta del mismo y en el sitio donante, con la consecuencia de disminuir la necrosis.

\section{Material y Método}

Este estudio fue realizado entre Enero de $2000 \mathrm{y}$ Diciembre de 2008.

El colgajo de tensor de fascia lata fue utilizado para cubrir 17 úlceras por presión trocantéreas en 16 pacientes.

\section{Selección de pacientes}

Todos los pacientes portadores de úlcera por presión trocantérica.

La edad fluctuó entre 24 y 64 años, 14 son hombres y 2 mujeres.

La etiología del síndrome de postración fue paraplejia por trauma raquimedular en 12 casos, TEC grave en 3 casos y compromiso de conciencia secundario a intoxicación medicamentosa por benzodiazepinas en 1 caso. De los 16 pacientes, 14 recibieron tratamiento por espasticidad.

En 2 de 16 pacientes existe comorbilidad: Depresión, Desnutrición e HTA.
Tabla 1. Úlceras por presión trocantéricas tratadas con colgajo de Tensor de Fascia lata en V-Y

- Úlceras trocantéricas: 17 en 16 pacientes

- Edad: 24 a 64 años

- Sexo: 14 hombres 2 mujeres

- Paraplejia: 12 pacientes

- TEC grave: 3

- Intoxicación por benzadiazepinas: 1

- Depresión, Desnutrición, Hipertensión arterial: 2

- 7 derechas

- 8 izquierdas

- 1 bilateral

- 5 pacientes úlceras grado III

- 11 pacientes úlceras grado IV

De las 17 úlceras por presión trocantéreas, 7 fueron derechas, 8 izquierdas y en un paciente fue bilateral. En 5 casos la úlceras por presión fueron de grado III y en 11 pacientes de grado IV (Tabla 1).

El tamaño de la úlcera fluctuaba entre los 3 y los $20 \mathrm{~cm}$ de diámetro.

En 14 pacientes coexistían otras úlceras, en 8 pacientes de localización sacras y en 6 isquiáticas.

\section{Técnica Quirúrgica}

El colgajo clásico del músculo tensor de fascia lata se basa en los vasos perforantes de dicho músculo, teniendo presente que la porción distal es fasciocutánea. Su irrigación proviene de la rama terminal de la arteria circunfleja femoral lateral, rama de la femoral profunda ${ }^{6}$.

Su inervación sensitiva es a través de ramos de $\mathrm{L}_{1}, \mathrm{~L}_{2} \mathrm{y} \mathrm{L}_{3}$.

Uno de los principales inconvenientes de la técnica clásica es la tensión en el cierre primario.

Es por eso, que surge como una buena y práctica manera de resolver este problema, el colgajo del tensor de fascia lata en V-Y, en que la porción más proximal es miocutánea y la distal es fasciocutánea.

Se diseña un colgajo en $\mathrm{V}$ con irrigación por su base superior con vértice inferior, contactando el extremo posterior con la úlcera. Se avanza y rota el colgajo cubriendo completamente la úlcera (Figura 1). El cierre se hace en forma de Y.

Se reseca la cicatriz de la úlcera y toda la bursa hasta obtener un lecho vital, como es de regla en el manejo de las úlceras por presión.

Si existen dudas sobre los márgenes de la bursa, puede realizarse una tinción con azul de metileno por todos los bordes de la cavidad. Así, al resecar, no deberá quedar rastro del azul en el lecho rema- 


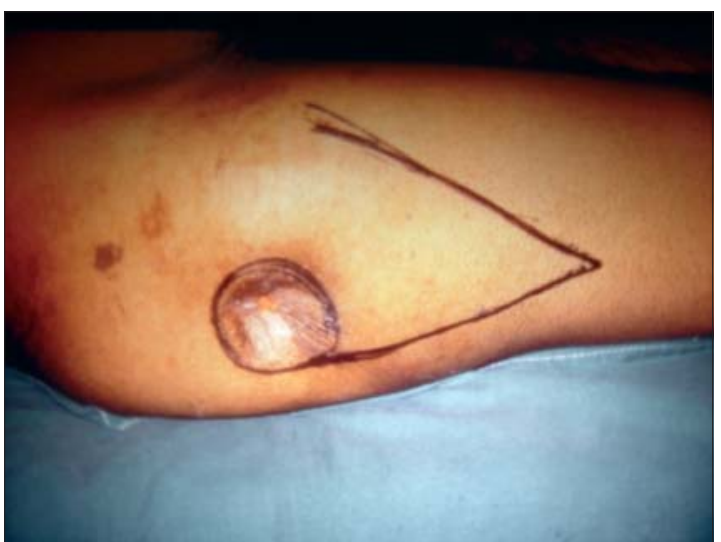

Figura 1. Úlcera trocantérica izquerda. Diseño del colgajo de tensor de la fascia lata en hacha.

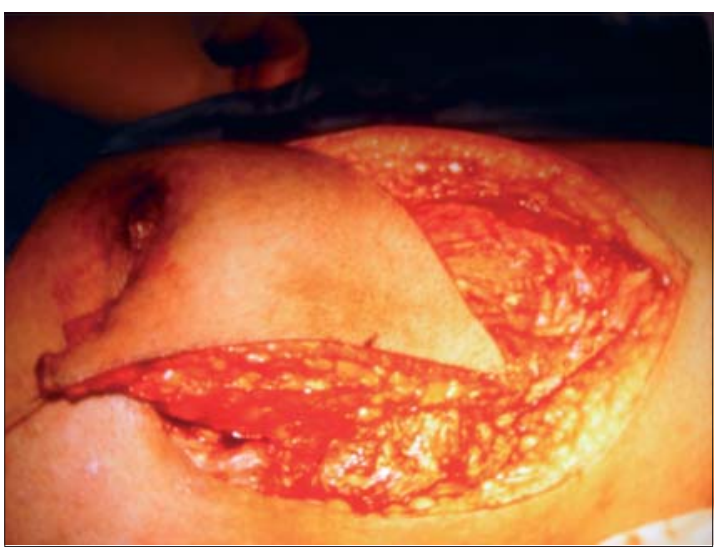

Figura 3. Colgajo en hacha cubriendo la úlcera por presión trocantérica.

nente. Se reseca la prominencia ósea, la que con cierta frecuencia presenta ostiomielitis, con cincel. La ostiomielitis la hemos diagnosticado con resonancia magnética.

El trozo de hueso se envía a Anatomía Patológica y a cultivo cuantitativo.

Se rota este colgajo cubriendo completamente la úlcera. El defecto dejado se cierra en Y (Figura 2). Se logra un cierre sin tensión de la zona dadora, así como una adecuada cobertura de la úlcera por presión.

Se deja un drenaje aspirativo en el lecho cruento bajo el colgajo, el que será retirado de acuerdo al débito cuantificado diariamente en no menos de 10 días (Figuras 3 y 4). La cicatrización se obtiene a los 21 días (Figura 5).

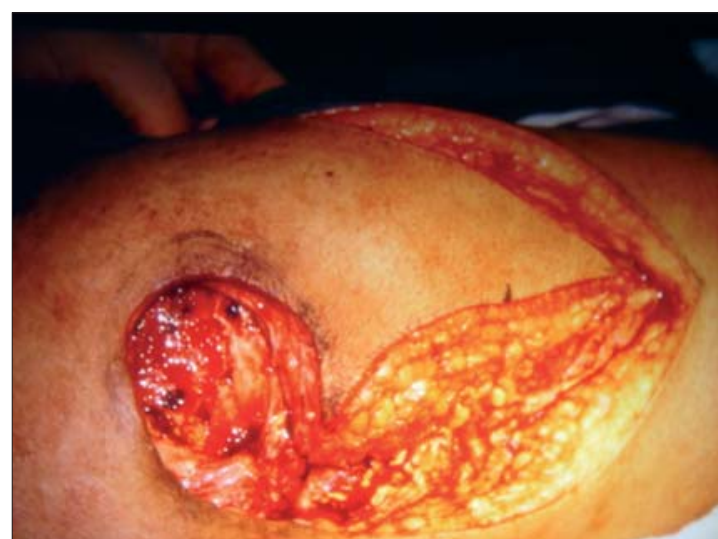

Figura 2. Elevación del colgajo en hacha.

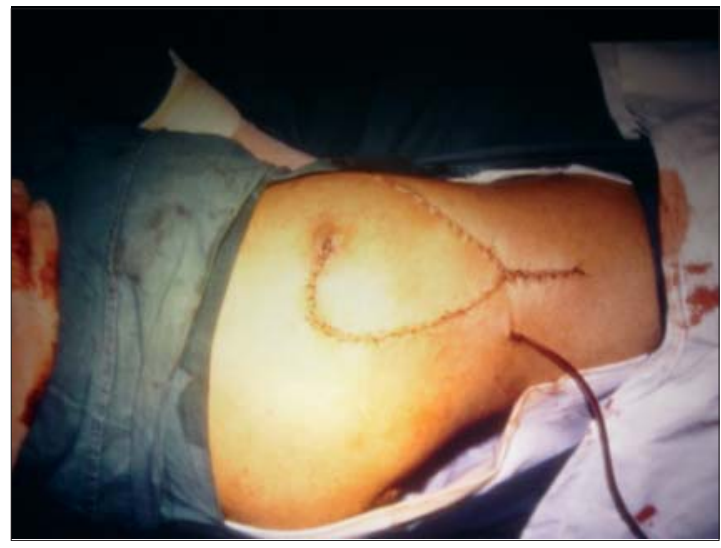

Figura 4. Colgajo en hacha. Resultado de la cobertura.

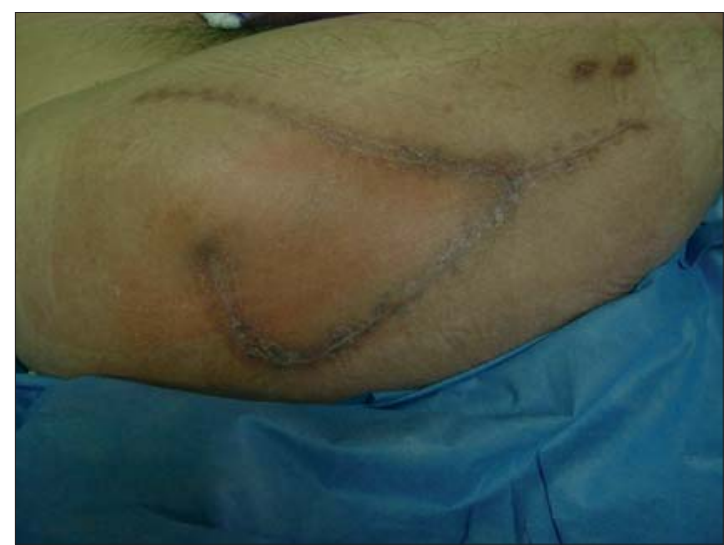

Figura 5. Resultado a largo plazo del colgajo en hacha. 


\section{Resultados}

Hubo seromas en 8 pacientes, resueltos con doble aspiración negativa (una externa y otra interna al colgajo); hubo dehiscencia de sutura en 7 pacientes, los que fueron resuturados; observamos infección en 6 pacientes, en los que se cultivó Proteus Mirabilis, SAMR, Klebsiella Pneumoniae, siendo tratados con aseos quirúrgicos. En 5 colgajos hubo necrosis parcial de la punta del colgajo que implicó resección y sutura. No hubo pérdida completa de colgajos. El tiempo de aparición de la complicación fluctuó entre el $3^{\circ}$ y los 55 días.

La resonancia magnética del trocánter demostró, en el preoperatorio, osteomielitis en 4 pacientes.

El drenaje fue retirado entre los $10 \mathrm{y}$ los 15 días. El sitio donante no requirió cobertura con injerto.

Se evidencia recidiva de la úlcera por presión en 2 pacientes a los 3 y 6 meses respectivamente, siendo reoperados con colgajo de rotación de músculo recto femoral.

Las reoperaciones fueron complementadas con VAC (Vacuum Assisted Closure) en 5 casos.

Los pacientes fueron dados de alta entre 31 a 150 días del postoperatorio.

El seguimiento fue de hasta 1 año postoperatorio.

\section{Discusión}

Se han propuesto múltiples modificaciones de la técnica del clásico colgajo tensor de fascia lata desde su introducción por Nahai en 1978. Una modificación es el avance en V-Y, que permite un adecuado cierre del defecto, minimizando la 'oreja de perro'. Lynch describió el colgajo tensor de fascia lata bilobulado, liberando de tensión la línea de sutura.

En este artículo se propone una modificación a la técnica clásica con el colgajo de tensor de fascia lata, en los pacientes con úlceras por presión trocantéreas, minimizando el defecto estético y funcional de la zona dadora y minimizado el sufrimiento de la punta del colgajo distal. La forma del colgajo está tomada de un colgajo en $\mathrm{V}-\mathrm{Y}^{7}$.
Si bien la literatura describe una tasa de recurrencia con el colgajo de tensor de fascia lata del $80 \%$ en úlceras por presión trocantéreas, este trabajo tiene recidiva en sólo 2 pacientes. En caso de necrosis parcial del colgajo, hemos resecado lo pertinente y rotado un colgajo tipo Cone Flap ${ }^{8}$.

Como conclusión, teniendo en cuenta el bajo número de complicaciones y su fácil resolución, sin pérdida completa del colgajo en esta serie, es que nos permitimos recomendarlo como una alternativa segura y de fácil realización para resolver este tipo de patología, que afecta a un número importante de pacientes.

\section{Referencias}

1. Bauer J, Mancoll JS, Phillips L. Pressure Sore. In Grabb \& Smith Plastic Surgery 6th Edition p. 722-729 Lippincott, Williams \& Wilkins. 2007.

2. Nahai F, Silverton JS, Hill HL. The Tensor Fasciae Lata Musculocutaneous Flap. Ann Plast Surg 1978; 1: 371-379.

3. Paletta CE, Freedman B, Shehadi SI. The Tensor Fasciae Latae Musculocutaneous Flap. Plast Reconstr Surg 1989; 83: 852-859.

4. Lynch SM. The Bilobed Tensor Fasciae Latae Myocutaneous Flap. Plast Reconstr Surg 1981; 67: 796-803.

5. Ishida L, Munhoz A, Montag E, Helio RN, Lopes S, Nakamoto HA, et al. Tensor Fasciae Latae Perforator Flap: Minimizing Donor-Site Morbidity in The Treatment of Trochanteric Pressure Sores. Plast Reconstr Surg 2005; 116: 1346-1352.

6. Mathes J, Nahai F. Clinical applicatios for muscle and musculocutaneous flaps. The C.V. Mosby Company. St Louis. Toronto. London 1982: 20-23.

7. Tuncali D, Barutcu AY, Gokrem S, Terzioglu A, Aslan G. The Hatchet Flap for Reconstruction of Fingertip Amputations. Plast Reconstr Surg 2006; 117: 19331939.

8. Calderón W, Andrades P, Cabello R, Israel G, Leniz P. The cone flap a new and versatile fasciocutaneuos flap. Plast Reconstr Surg 2004; 114: 1539-1542. 